\title{
Portfolios Come Full Circle in the World of Work
}

Janine Quiding

Waitematā District Health Board

Miriam Laidlaw

Waitematā District Health Board

Tabitha Parker

Waitematā District Health Board

Kristina Hoeppner

Catalyst

kristina@catalyst.net.nz

Submission Type: Case Study (20mins + 10minsQ\&A)

Keywords: ePortfolio, Healthcare, Professional development, Lifelong learning

\section{Abstract:}

Electronic portfolios become increasingly visible in the workplace. Students graduate with portfolios and are in a good position to continue with portfolio practices. Additionally, organizational or professional competency frameworks and registration requirements lend themselves to be evidenced by portfolios. Waitematā District Health Board, the largest in Aotearoa New Zealand, piloted an electronic portfolio at the end of 2018 with 90 nurses and started implementing the new ePortfolio organisation-wide for nurses in 2019.

In this presentation, we will provide an overview of the pilot and position it within the organisation's commitment to learning and development. We will outline the advantages for all involved: nurses, peers, managers, and assessors, and discuss the challenges and future directions for the initiative.

Early results indicate that the portfolios allow for a shift in conversation with staff and in the development of practice. They encourage nurses to consider additional training and studies to advance in their careers and have the evidence available to discuss with their manager to set clear goals.

The nurses go full circle: They started out with their studies, became professionals, and are going back to university for postgraduate studies documenting their learning in portfolios along the way. Portfolios in this context support lifelong learning and make that learning visible to the individual nurses and their organisation. 